\title{
Acute Hydronephrosis owing to A Giant Fecaloma in an Older Patient
}

\author{
Narae Joo ${ }^{1}$, Hyung Seok Lee ${ }^{2}$ \\ ${ }^{1}$ Department of Nephrology, Hanmaeum General Hospital, Jeju, Korea \\ ${ }^{2}$ Division of Nephrology, Department of Internal Medicine, Hallym University Sacred Heart Hospital, Anyang, Korea
}

Corresponding Author:

Hyung Seok Lee, MD

Division of Nephrology, Department of Internal Medicine, Hallym University

Sacred Heart Hospital, 22

Gwanpyeong-ro 170beon-gil, Dongan-

gu, Anyang 14068, Korea

E-mail: pcsacred@gmail.com

ORCID:

https://orcid.org/0000-0001-6380-9243

Received: August 8, 2020

Revised: September 4, 2020

Accepted: September 5, 2020
Hydroureteronephrosis with acute urinary tract obstruction can lead to serious complications such as obstructive nephropathy or pyelonephritis. We report the first case of hydroureteronephrosis caused by a giant fecaloma in an 83-year-old woman accompanied with chronic constipation in Korea. The patient with a fever presented to the emergency room and was diagnosed with right hydronephrosis. Computed tomography revealed a markedly dilated rectum with a giant fecaloma compressing the right vesicoureteric junction. Hydroureteronephrosis was resolved through manual disimpaction of the fecaloma and simultaneous lavage with rectal tubes, which improved the acute pyelonephritis and renal function. Chronic constipation can lead to giant fecaloma threatening urinary tract patency, especially in older patients with immobility or dehydration. Therefore, clinicians should consider this condition as a rare but possible cause of hydronephrosis in older patients with chronic constipation.

Key Words: Hydronephrosis, Fecal impaction, Elderly

\section{INTRODUCTION}

Acute urinary tract obstruction can cause acute kidney damage and urinary tract infections, which can lead to potentially fatal complications. Although rare, acute urinary tract obstruction can be caused by compression of the gastrointestinal (GI) tract. Some cases of acute urinary tract obstruction by giant fecalomas caused by chronic constipation have been reported in patients with immobility and dehydration. ${ }^{1)}$ In addition, constipation and fecal impaction are common clinical problems in older adults. ${ }^{2)}$

Even with its clinical relevance, chronic constipation is rarely suspected as a predisposing cause of urinary tract obstruction. To date, there have been no reports of fecaloma-related acute hydronephrosis owing to chronic constipation in Korea. Here, we report a case of acute hydronephrosis owing to a giant fecaloma in an older patient.

\section{CASE REPORT}

An 83-year-old woman with a fever presented to the emergency room. She had a 10-year history of chronic constipation and was in a long-term bedridden state owing to a previous history of right femur fracture, cerebral infarction, and dementia. She had no history of abdominal surgery, inflammatory bowel disease, or anatomical abnormalities of the GI tract. Her blood pressure was $130 / 70 \mathrm{mmHg}$; heart rate, $81 / \mathrm{min}$; respiratory rate, $22 / \mathrm{min}$; and body temperature, $37.8^{\circ} \mathrm{C}$. The patient was mentally alert but was not able to follow instructions owing to severe dementia. She had a history of cerebral infarction with neurologic sequelae of left hemiplegia and was in a bedridden state owing to a right femur fracture following a fall that occurred 1 month earlier.

Physical examination of the patient revealed diffuse abdominal tenderness, and a firm and huge mass was palpable between her pubic bone and umbilicus. She had no rebound tenderness. Bowel sounds were hypoactive. Blood test results were as follows: white blood count, $8,700 / \mu \mathrm{L}$ with neutrophilia (83.4\% white blood cells [WBCs]); hemoglobin, $11.7 \mathrm{~g} / \mathrm{dL}$; and C-reactive protein, 65.3 $\mathrm{mg} / \mathrm{L}$ (markedly increased). Electrolytes and thyroid function test results were normal. Proteinuria $2+$, hematuria $3+$ (red blood cell $>100$ in microscopic examination), and pyuria (WBC count 50$99 / \mu \mathrm{L}$ in microscopic examination) were detected following the 
urine analysis.

Serum blood urea nitrogen (BUN) and creatinine concentrations were mildly increased to $43.0 \mathrm{mg} / \mathrm{dL}$ and $0.93 \mathrm{mg} / \mathrm{dL}$, respectively. Blood and urine microbiological cultures grew Escherichia coli sensitive to all tested antibiotic agents. A Foley catheter was introduced into the urinary bladder, and the remaining urine volume was $50 \mathrm{~mL}$. Computed tomography revealed a markedly dilated rectum with a giant fecaloma compressing the right lower ureter and vesicoureteric junction, resulting in right hydronephrosis (Fig. 1). Through the manual release of the fecaloma and simultaneous enema with rectal tubes, fecal impaction and rectal dilatation were resolved. Subsequently, hydroureteronephrosis was resolved, which was confirmed by ultrasonography (Fig. 2). Acute pyelonephritis and renal function improved and the patient's BUN and serum creatinine concentrations decreased to $19.6 \mathrm{mg} / \mathrm{dL}$ and $0.69 \mathrm{mg} / \mathrm{dL}$, respectively. In addition, the serum C-reactive protein concentration decreased to $7.77 \mathrm{mg} / \mathrm{L}$. The patient was discharged in good medical condition with normal renal and bowel functions.

The present case report was reviewed by the Institutional Review Board of Hallym University Sacred Heart Hospital (No. HALLYM 2020-05-011), and informed consent was obtained from the patient for publication.

\section{DISCUSSION}

Fecal impaction can be caused by GI tract damage owing to Hirschsprung disease, prior intestinal surgeries, and anorectal malformations. ${ }^{3-5)}$ However, chronic constipation is also a common cause of fecal impaction, especially in older adults. ${ }^{6)}$ A fecaloma is a mass of inspis-

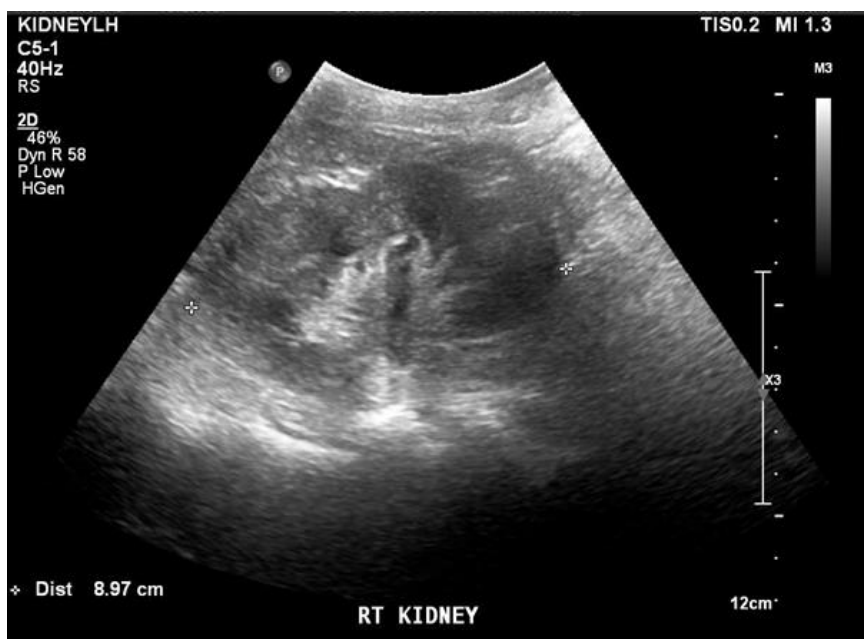

Fig. 2. Kidney ultrasound demonstrating a longitudinal view of normalsized kidneys and a shape with the resolution of hydronephrosis in the right kidney.
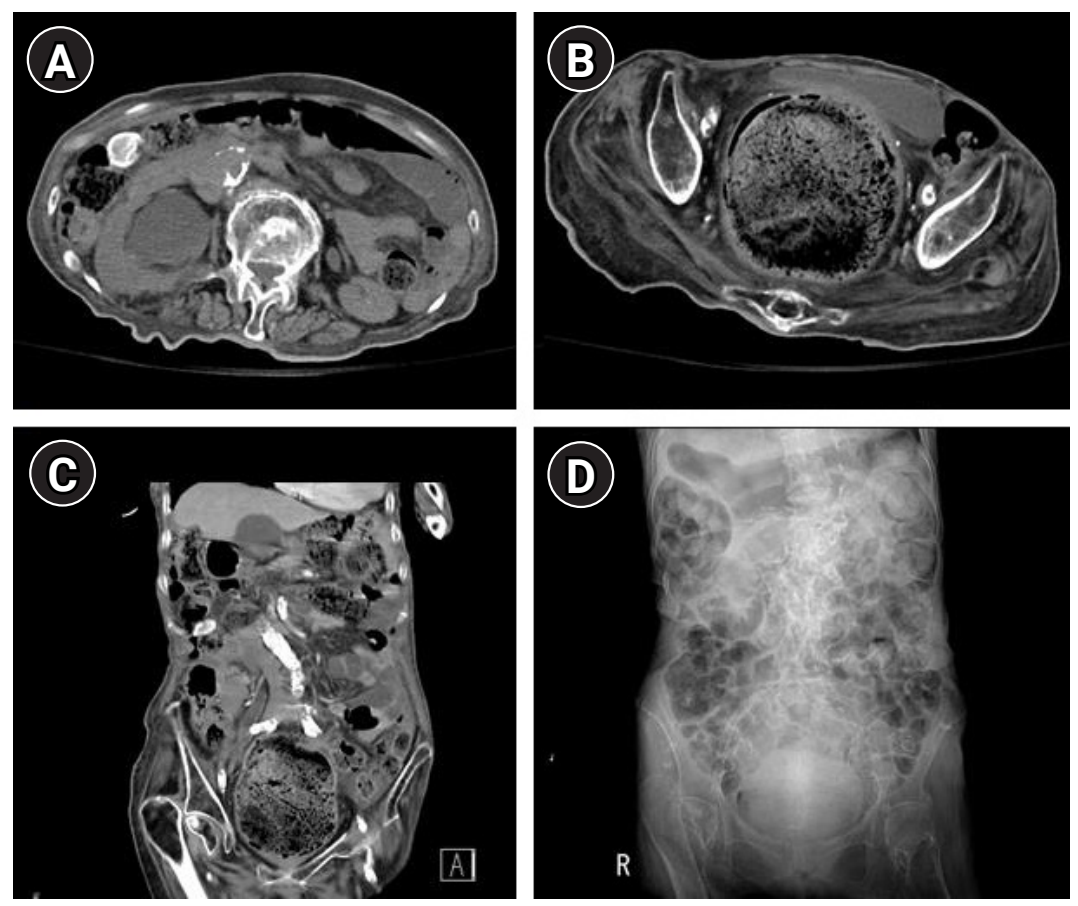

Fig. 1. Hydroureteronephrosis caused by a giant fecaloma in the rectum. (A) Computed tomography (CT) of the abdomen-pelvis showing hydronephrosis in the right kidney. (B) CT of the abdomen-pelvis showing a huge distended rectum containing a giant fecaloma pushing the urinary bladder anteriorly and compressing the vesicoureteral junction. (C) CT of the abdomen-pelvis showing right hydroureter owing to a markedly dilated rectum caused by the huge fecaloma. (D) Giant fecaloma is visible in the kidney-ureter-bladder image. 
sated stool that results from the accumulation of feces in the rectum or rectosigmoid colon. Fecalomas are much harder in consistency than a fecal impaction and can cause a mass effect that compresses adjacent anatomical structures, leading to potentially fatal complications unless appropriately treated. ${ }^{1,7)}$ In the literature, fecalomas can reportedly lead to bowel ischemia, bowel perforation, and peritonitis. ${ }^{7,8)}$ However, obstructive nephropathy with compression by fecalomas owing to chronic constipation is rarely reported. ${ }^{9,10)}$ Fecaloma can result in a marked dilatation of the rectosigmoid colon, subsequently leading to extrinsic ureteral compression and acute urinary tract obstruction owing to anterior displacement of the bladder base. ${ }^{11)}$ Bedridden patients with immobility owing to neurological or traumatic sequelae are at a higher risk of developing urinary tract obstruction caused by chronic constipation-induced fecalomas, especially if they are superimposed by insufficient fluid intake, dehydration, or administration of drugs that decrease GI tract motility. ${ }^{12-14)}$ Moreover, patients with schizoaffective disorders who are receiving antipsychotic, anticholinergic, tricyclic antidepressant, and antiserotonergic medication are also at a higher risk. ${ }^{12-15)}$ Consistent with these reports, our patient was also bedridden owing to a previous cerebral infarction and femur fracture; she also had a history of chronic constipation. Moreover, her medical records confirmed that the patient had been taking analgesics for the femur fracture. Because it was difficult to communicate with the patient owing to advanced dementia, we assumed that she could not complain about her symptoms until hydronephrosis resulting from the giant fecaloma occurred.

Besides unilateral or bilateral acute kidney injury, urinary tract obstruction caused by giant fecalomas can lead to acute pyelonephritis or emphysematous pyelonephritis; therefore, urgent treatment, including the removal of the cause, is necessary. ${ }^{16)}$ Most fecal impactions are successfully treated by conservative methods such as laxatives, manual disimpaction, including digital evacuation, or transrectal enemas. ${ }^{17,18)}$ In cases refractory to conservative methods, an endoscopic approach for removing fecalomas has also been described. ${ }^{19,20)}$ Surgery is required only in severe cases. ${ }^{15)}$ Fortunately, conservative treatment with rectal enema and digital evacuation was successful in our patient.

Although extremely rare, fecal impaction should be considered in the differential diagnosis of acute obstructive nephropathy, especially in older patients with limited mobility, history of cerebrovascular accidents, and intake of agents that decrease bowel movements. Chronic constipation can lead to giant fecaloma threatening urinary tract patency, especially in older people with immobility and dehydration or those who are taking analgesics that decrease GI motility. Although giant fecalomas are rare causes of acute obstructive nephropathy or pyelonephritis, physicians should consid- er them as possible causes of hydronephrosis in older patients with chronic constipation.

\section{ACKNOWLEDGMENTS}

\section{CONFLICT OF INTEREST}

The researchers claim no conflicts of interest.

\section{AUTHOR CONTRIBUTIONS}

Conceptualization, HSL; Data curation, NJ, HSL; Investigation, NJ, HSL; Methodology, NJ, HSL; Project administration, HSL; Supervision, HSL; Writing-original draft, NJ, HSL; Writing-review \& editing, NJ, HSL.

\section{REFERENCES}

1. Caiazzo P, De Martino C, Del Vecchio G, Di Lascio P, Marasco M, Laviani F, et al. Megacolon for a giant faecaloma with unlucky outcome: case report and review of the literature. Ann Ital Chir 2013;84:319-22.

2. Oh SH. Constipation in the elderly. J Korean Geriatr Soc 2001; 5:209-17.

3. Altomare DF, Rinaldi M, Sallustio PL, Armenise N. Giant fecaloma in an adult with severe anal stricture caused by anal imperforation treated by proctocolectomy and ileostomy: report of a case. Dis Colon Rectum 2009;52:534-7.

4. Cheng M, Ghahremani S, Roth A, Chawla SC. Chronic constipation and its complications: an interesting finding to an otherwise commonplace problem. Glob Pediatr Health 2016;3: 2333794.X16648843.

5. Park JS, Park TJ, Hwa JS, Seo JH, Park CH, Youn HS. Acute urinary retention in a 47-month-old girl caused by the giant fecaloma. Pediatr Gastroenterol Hepatol Nutr 2013;16:200-5.

6. Serrano Falcon B, Barcelo Lopez M, Mateos Munoz B, Alvarez Sanchez A, Rey E. Fecal impaction: a systematic review of its medical complications. BMC Geriatr 2016;16:4.

7. Celayir MF, Koksal HM, Uludag M. Stercoral perforation of the rectosigmoid colon due to chronic constipation: a case report. Int J Surg Case Rep 2017;40:39-42.

8. Ouaissi M, Sielezneff I, Benoist S, Pirro N, Cretel E, Chaix JB, et al. Lethal fecaloma.J Am Geriatr Soc 2007;55:965-7.

9. Perfecto Valero A, Serrano Hermosilla C, Aranda Escano E, Tellaeche de la Iglesia M. Right hydronephrosis secondary to fecal impaction. Cir Esp 2019;97:407.

10. Hernandez Garcia E, Torres Sanchez MJ, Gomez Sanchez J. Fecaloma as a cause of acute renal failure. Rev Clin Esp 2019;219: 462-3. 
11. Kerrigan DD, Lucas MG, Sun WM, Donnelly TC, Read NW. Idiopathic constipation associated with impaired urethrovesical and sacral reflex function. Br J Surg 1989;76:748-51.

12. Knobel B, Rosman P, Gewurtz G. Bilateral hydronephrosis due to fecaloma in an elderly woman. J Clin Gastroenterol 2000; 30:311-3.

13. Tu KC, Kuo JR. Fecaloma causing megacolon and bilateral hydronephrosis. Formos J Surg 2020;53:70-3.

14. Kim MY, Etherton-Beer C, Kim CB, Yoon JL, Ga H, Kim HC, et al. Development of a Consensus List of Potentially Inappropriate Medications for Korean Older Adults. Ann Geriatr Med Res 2018;22:121-129.

15. Khan MA, Dar HA, Shah AH, Javid G, Singh B, Sheikh NA, et al. Fecaloma presenting as huge abdominal mass. JGH Open
2019;4:294-5.

16. Abi Abdallah M, Raad N, Yarak N, Noujeim JP, Noujeim A. Emphysematous pyelonephritis caused by a giant fecaloma. Case Rep Urol 2019;2019:8743525.

17. Curro G, Lazzara C, Latteri S, Bartolotta M, Navarra G. Supergiant fecaloma as manifestation of chronic constipation. G Chir 2017;38:53-4.

18. Yamasaki M, Funaishi K, Hattori N. Giant fecaloma causing anorexia and dysuria. Clin Gastroenterol Hepatol 2020;18:A30.

19. Ghosh G, Shah S, Maltz C. A case of a giant fecaloma. Clin Gastroenterol Hepatol 2018;16:e48.

20. Sakai E, Inokuchi Y, Inamori M, Uchiyama T, Iida H, Takahashi $\mathrm{H}$, et al. Rectal fecaloma: successful treatment using endoscopic removal. Digestion 2007;75:198. 